\title{
EXPERIMENTAL MODELLING OF VIBROACOUSTICS OF THE HUMAN VOCAL TRACT WITH COMPLIANT WALLS
}

\author{
Radolf V.:, Horáček J., Košina J.
}

\begin{abstract}
Experimental model of human vocal tract cavities with hard walls has been modified to take into account the compliance of the soft tissue of the human vocal tract. The paper presents the studied acousticstructural interaction of the vocal tract cavities with a dynamical system originated in vibration of the soft tissue. The experimental results are in qualitative agreement with the results of mathematical modelling. Compliant walls of acoustic cavities generate additional low frequency acoustical-mechanical resonances of the system and increase acoustic resonance frequencies.
\end{abstract}

Keywords: Biomechanics of voice, Vocal tract acoustics, Acoustic-structural interaction.

\section{Introduction}

Human voice production is a complex physiological process. Airflow coming from the lungs induces selfoscillating vocal fold vibration generating a primary sound. This sound is then modified in the airways above the vocal folds, i.e. in the vocal tract (VT). The frequency spectrum of the resulting sound is thus affected by acoustic resonances of VT cavities revealing so-called formants - peaks in the envelope of the voice spectrum, see e.g. Sundberg (1987). Formant frequencies define vowels and the voice timbre. Both frequency and amplitude of formants can be influenced by the compliance of the soft tissues of vocal cavities. According to the numerical simulations and measurements on humans (Radolf et al., 2016), this influence is evident mainly for the lowest formant frequencies. Experimental simulations of this phenomenon can help to study in detail acoustic-structural interaction of the VT cavities with a dynamical system originated in mechanical resonances of the soft tissue.

\section{Methods}

A vocal tract model with cross-sectional rectangular areas was made of hard material plexiglass. Its main geometric configuration was taken from a 3D volume model obtained from magnetic resonance images for the Czech vowel [u:], see Vampola et al. (2008). Subsequently the upper wall of the VT model was replaced with a soft membrane made of silicone rubber Ecoflex ${ }^{\mathrm{TM}} 00-50$, see Fig. 1. The thickness of the membrane was $1 \mathrm{~mm}$, and it was slightly prestressed during the fastening to other parts of the VT model.

\subsection{Identification of the vibration characteristic of the compliant wall}

Resonance properties of the compliant wall at the top of the VT model were examined using the excitation by a loudspeaker $(170 \mathrm{~mm}$ in diameter, $8 \Omega, 150 \mathrm{~W})$ mounted on a plastic tube of diameter $160 \mathrm{~mm}$ and the length of $1.05 \mathrm{~m}$. The open end of the tube focused the acoustic waves on the compliant VT wall in the form of a unidirectional linear swept harmonic signal in the range from $10 \mathrm{~Hz}$ to $210 \mathrm{~Hz}$ with a rate $50 \mathrm{~Hz} / \mathrm{s}$ and a duration of $16 \mathrm{~s}$. The entrance to the VT model was closed by a piece of plasticine. A B\&K 2239 sound level meter recording a reference exterior signal was installed close to the top of VT model and directed at the loudspeaker. A Polytec OFV-505 laser vibrometer with an OFV-5000 controller was used for measuring vibration of the silicone membrane at the mouth cavity level, i.e. in the position of the cheek in the human vocal tract, and $4 \mathrm{~cm}$ from the VT input, i.e. at the end

\footnotetext{
* Ing. Vojtěch Radolf, PhD., Ing. Jaromír Horáček, DSc., Ing. Jan Košina: Institute of Thermomechanics of the Czech Academy of Sciences, Dolejškova 1402/5, 18200 Prague, Czech Republic, radolf@it.cas.cz; jaromirh@it.cas.cz; kosina@it.cas.cz
} 
of the epilaryngeal tube model. Transfer functions of the membrane vibration velocity to the reference acoustic pressure signal were measured in frequency domain.

\subsection{Identification of the acoustical - mechanical characteristic of the system}

Excitation of acoustic cavities of the VT model was realized by a small speaker ( $40 \mathrm{~mm}$ in diameter, $32 \Omega, 0.1 \mathrm{~W})$ placed and sealed in the input of the vocal tract, i.e. instead of the vocal folds, see Fig. 1. A unidirectional linear swept harmonic signal in the range from $150 \mathrm{~Hz}$ to $5155 \mathrm{~Hz}$ with a rate $715 \mathrm{~Hz} / \mathrm{s}$ was exciting acoustic waves in the VT model for the duration of $14 \mathrm{~s}$. For acoustic analysis of the system in the low-frequency range excitation, the swept harmonic signal from $10 \mathrm{~Hz}$ to $210 \mathrm{~Hz}$ with a rate $50 \mathrm{~Hz} / \mathrm{s}$ and a duration of $16 \mathrm{~s}$ was used. Frequency characteristic of the speaker was measured in a free space (without the VT model). Since this characteristic is rather flat, i.e. without resonances in the measured frequency range, it was not taken into consideration in subsequent data processing.

The acoustic pressure was measured $3 \mathrm{~mm}$ inside the mouth of the VT model with a special B\&K 4182 microphone probe (range $1 \mathrm{~Hz} \div 20 \mathrm{kHz}$ ). The acoustic signal outside the vocal tract model was recorded with a B\&K 2239 sound level meter. A laser vibrometer was used for measuring vibration of the soft VT wall in the same manner as described above.

All the measured signals were simultaneously sampled at a frequency of $16.384 \mathrm{kHz}$ or $4096 \mathrm{~Hz}$ for the low-frequency range and registered by a Brüel \& Kjaer PULSE type $3560 \mathrm{C}$ measurement system controlled by a personal computer equipped with SW PULSE LabShop Version 10.

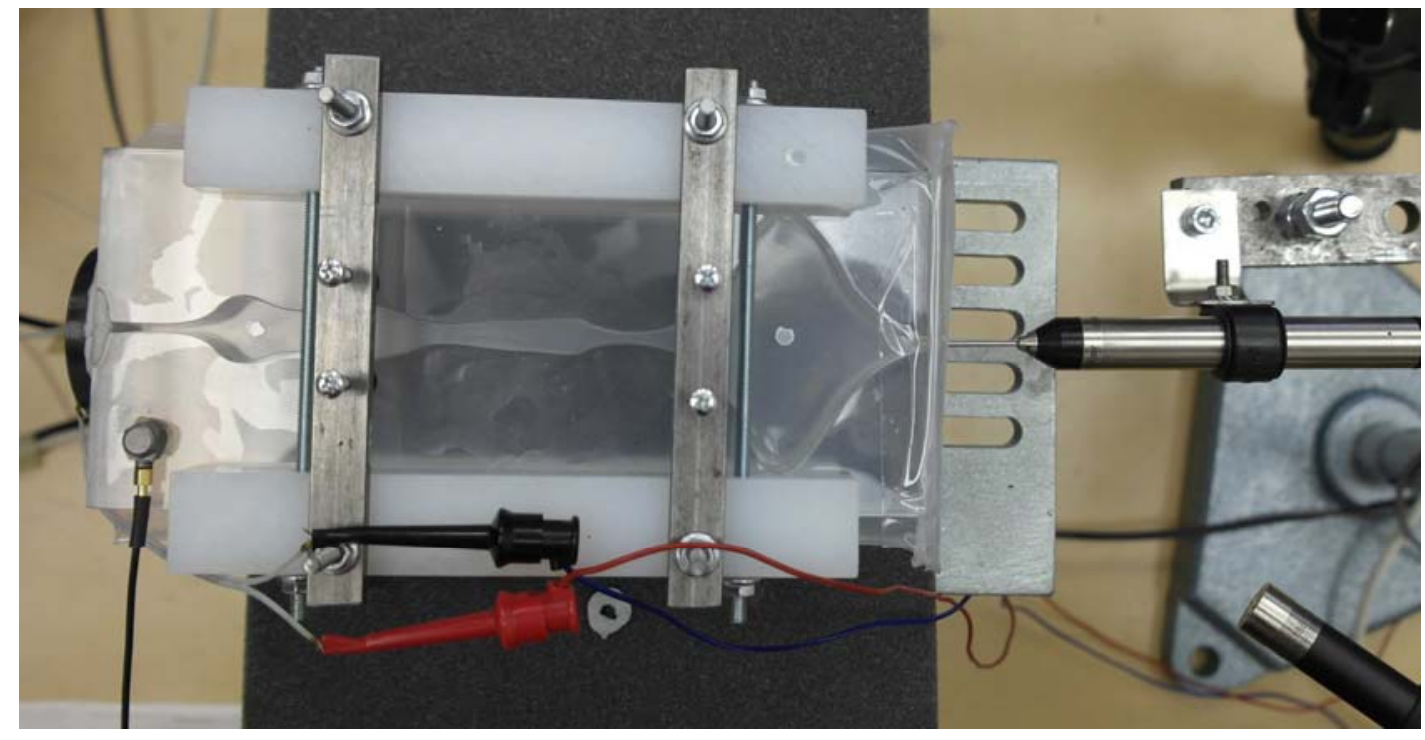

Fig. 1: Measurement set-up for excitation of the vocal tract model with the yielding wall by the speaker placed instead of the vocal folds. The speaker is situated on left side at the entrance to the vocal tract and the generated acoustic pressure was measured by the microphone probe at the oral cavity outlet and by external microphone placed in front of the vocal tract model.

\section{Results}

Spectra of the transfer function between the reference acoustic pressure signal and the membrane vibration velocity are shown in Fig. 2. Since the first acoustical-mechanical resonance frequency of $48 \mathrm{~Hz}$ was too close to common electromagnetic disturbance of $50 \mathrm{~Hz}$ originating in the electrical network, (see the dotted curve), the dynamical system was retuned. A ring-shaped piece of plasticine was added on the soft wall in the middle of the cheek model. This additional mass of $0.2 \mathrm{~g}$ decreased the first resonance to $40 \mathrm{~Hz}$ and increased its level (see the black solid line in Fig. 2), and it also reduced the levels of higher resonances.

Transfer function of the velocity, measured at the epilarynx position to the reference acoustic signal when the mass $0.2 \mathrm{~g}$ was added on the cheek, is depicted by the grey solid curve in Fig. 2. It reveals that in the low-frequency region up to about $90 \mathrm{~Hz}$, the energy of the vibrating wall is concentrated mainly in the cheek. For higher frequencies vibrations in the epilaryngeal region of the VT model become dominant. 


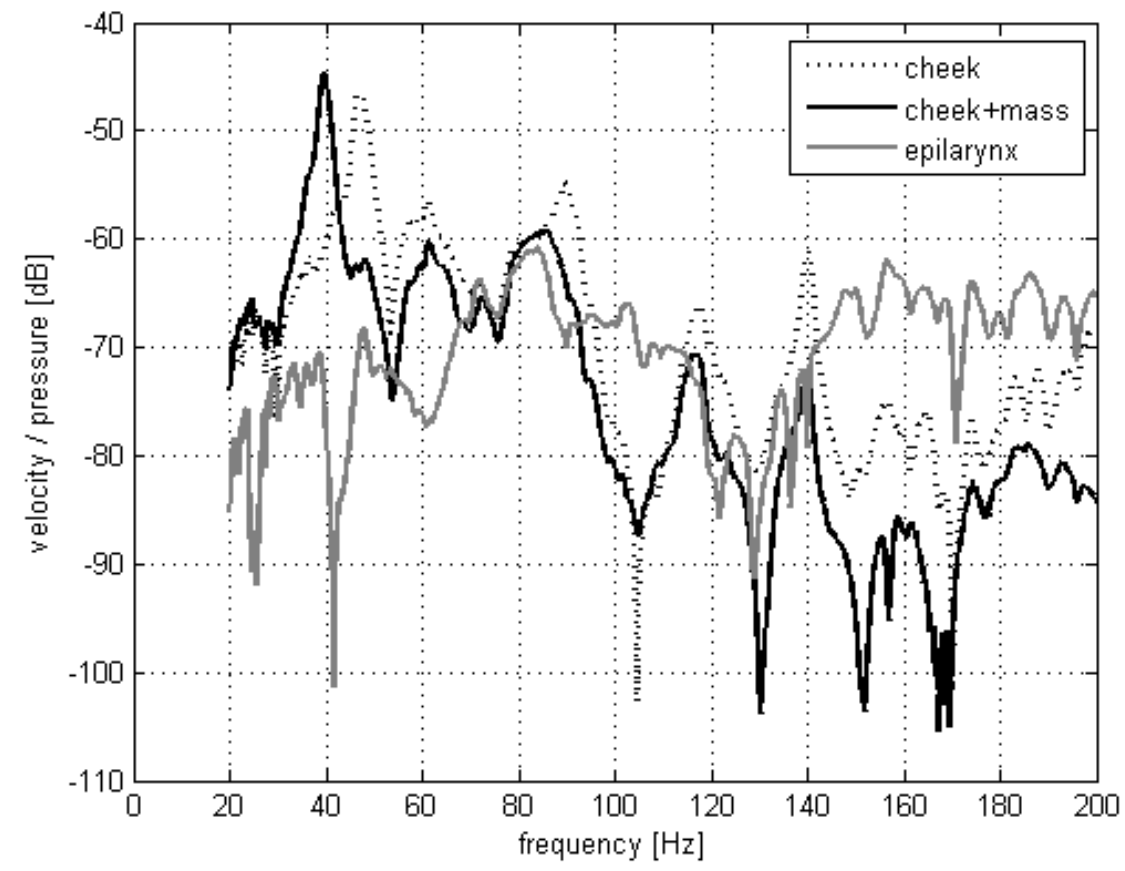

Fig. 2: Transfer functions between the reference acoustic signal and the compliant wall vibration velocity measured at the position of cheek (dotted curve), with added mass at the cheek (black solid curve) and at the epilarynx position (grey solid curve) with added mass at the cheek.

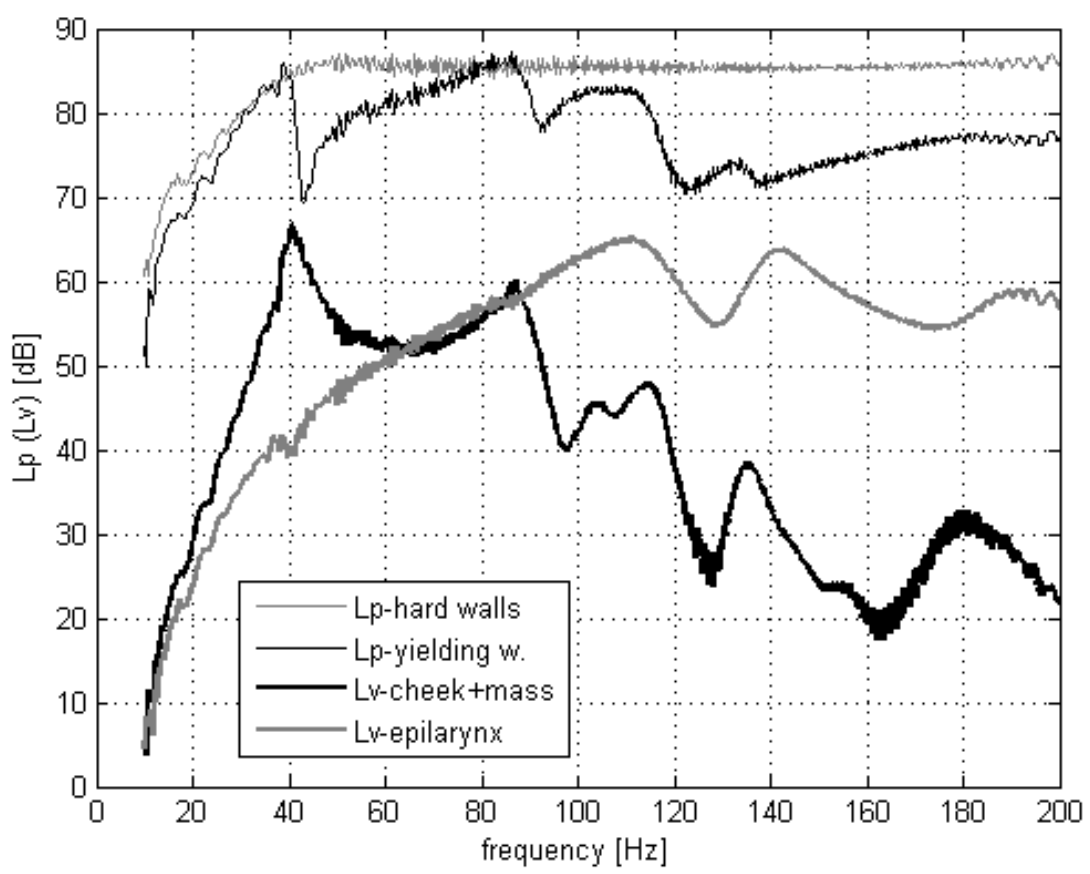

Fig. 3: Sound pressure spectra measured with microphone probe at the lips for hard-walled VT (grey thin curve) and yielding-walled VT (black thin curve), spectra of the velocity of vibrating yielding wall measured at the position of cheek (black thick curve) and at the position of the epilarynx in the VT model (grey thick curve).

For excitation of acoustic cavities by a small speaker placed in the input of the vocal tract, the spectra of the acoustic pressure measured in mouth and the velocity of the membrane vibration are compared with the spectra measured in the VT with the hard walls in Figs. 3 and 4 . The lowest mechanical resonances of the compliant wall, which belong to the cheek vibration (the black thick curve in Fig. 3), affect the acoustic pressure in the mouth cavity (black thin curve). The first mechanical resonance at $40 \mathrm{~Hz}$ causes a pressure spectral peak at $39 \mathrm{~Hz}$ and a spectral dip at $43 \mathrm{~Hz}$. Similar pattern can be seen also around the second mechanical resonance at $87 \mathrm{~Hz}$ and around the first mechanical resonance at the epilarynx at $110 \mathrm{~Hz}$ (grey thick curve). 


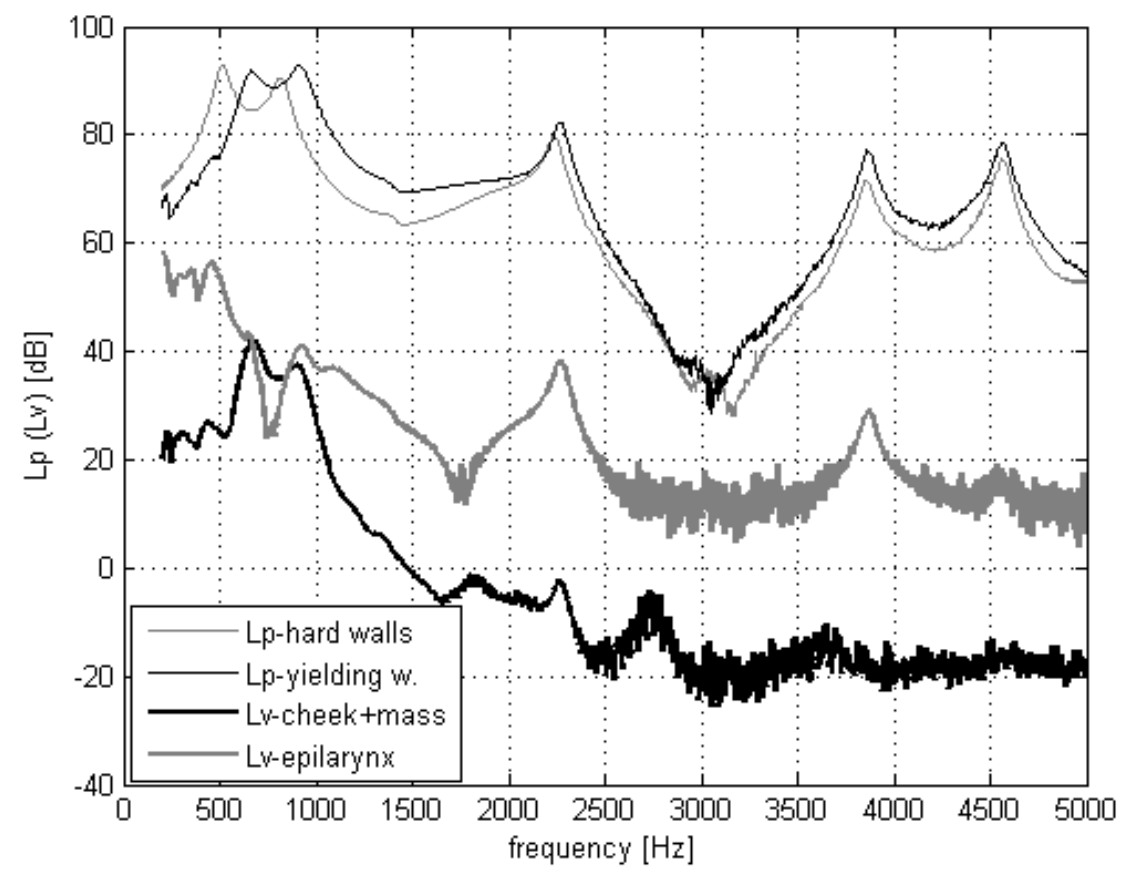

Fig. 4: Sound pressure spectra in high-frequency range measured with microphone probe at the lips for hard-walled VT (grey thin curve) and yielding-walled VT (black thin curve), spectra of the velocity of vibrating yielding wall measured at the cheek of the VT model (black thick curve) and at the epilarynx position (grey thick curve).

All acoustic resonance frequencies increased in case of compliant-walled VT model (see the black thin curve in Fig. 4) compared to the model with the hard walls (grey thin curve). The first and second acoustic resonances shifted significantly (by $30 \%$ and $12.1 \%$, respectively) towards higher frequencies. The higher three resonance frequencies increased only negligibly by $1.3 \%, 0.08 \%$ and $0.18 \%$. The first and the second acoustic resonance influence mainly vibration of the cheek (black thick curve in Fig. 4), whereas the third and the fourth acoustic resonances cause sharper peaks in the spectrum of the velocity at the epilaryngeal region of the VT model (grey thick curve in Fig. 4).

\section{Conclusion}

The novel experimental set-up has been designed to model acoustic-structural interactions of the vocal tract cavities with the soft tissue. Although the vibrating mass and the mechanical damping are lower compared to properties of human soft tissue (Hanna et al., 2016), the results are in qualitative agreement with the results of numerical simulations (Radolf et al., 2016). In future work the system should be tuned to increase both damping and mass of the vibrating wall, and full acoustic-flow-structure interaction will be studied when the self-oscillating vocal folds model excite the acoustic cavities covered with the compliant wall.

\section{Acknowledgement}

The study was supported by a grant from the Czech Science Foundation: No. 19-04477S "Modelling and measurements of fluid-structure-acoustic interactions in biomechanics of human voice production."

\section{References}

Hanna, N., Smith, J. and Wolfe, J. (2016) Frequencies, bandwidths and magnitudes of vocal tract and surrounding tissue resonances, measured through the lips during phonation. Journal of the Acoustical Society of America, 139, pp. 2924-2936.

Radolf, V., Horáček, J., Dlask, P., Otčenášek, Z., Geneid, A. and Laukkanen, A.M. (2016) Measurement and mathematical simulation of acoustic characteristics of an artificially lengthened vocal tract. Journal of Sound and Vibration, 366, pp. 556-570.

Sundberg, J. (1987) The science of the singing voice. DeKalb, Illinois: N. Illinois Univ. Press.

Vampola, T., Horáček, J. and Švec, J. G. (2008) FE modeling of human vocal tract acoustics. Part I: Production of Czech vowels. Acta Acustica united with Acustica, 94, pp. 433-447. 